\title{
WAVES AND STRONGLY SHEARED CURRENTS: EXTENSIONS TO COASTAL OCEAN MODELS
}

\author{
Saeideh Banihashemi, University of Delaware, bhashemi@udel.edu \\ James T. Kirby, University of Delaware, Kirby@udel.edu \\ Fengyan Shi, University of Delaware, fyshi@udel.edu \\ Zhifei Dong, University of Delaware, zdong@udel.edu
}

\section{OVERVIEW}

Significant progress has been made in the numerical modeling of wave-current interaction during the past decade. Typical coastal circulation and wave models, however, still only employ theoretical formulations which take depth-uniform mean flows into account, with realistic, non-uniform flows treated as being depthuniform through some chosen averaging procedure. Depending on the choice of average over depth, significant errors may arise in the estimation of properties such as group velocity and action density in realistic conditions. These errors, in turn, are fed back into the circulation model through incorrect representation of the vertical structure of wave forcing.

A new framework for wave-current interaction theory for strongly sheared mean flows has been developed using vortex force formalism by Dong (2016). The resulting formulation leads to a conservation law for wave action identical to that of Voronovich (1976), and to expressions for wave-averaged forces in the Craik-Leibovich vortex force formalism. In this study, we are completing the development of a coupled NHWAVE/SWAN which implements the wave forcing formulation of Dong (2016) in a wave-averaged version of the non-hydrostatic model NHWAVE (Ma et al., 2012). The SWAN model is also being extended to incorporate a better representation of frequency and direction-dependent group velocity and intrinsic frequency in the neighborhood of the spectral peak, thus improving on the present practice of using quantities evaluated only at the spectral peak. The resulting model is being tested against field data collected in several recent experiments involving strong, vertically sheared currents in river mouths or straits.

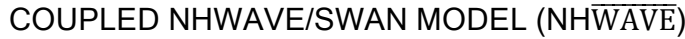

Dong (2016) derived a general wave/current interaction theory in a form analogous to that of McWilliams et al (2004), but extended to include effects of arbitrarily strong vertical shear in ambient current profiles. The vortex force formulation from Dong's theory was implemented in a coupled NHWAVE/SWAN model, denoted NHWAVE. In this study, we are extending Dong's theory to incorporate spectral wave conditions in order to generalize the model for realistic field conditions.

\section{WAVE MODEL MODIFICATIONS}

Banihashemi et al., (2017, BKD17) demonstrate that the widely used depth-weighted average current value (Kirby \& Chen,1989, KC89) is not the correct current speed to use directly in the action equation in SWAN or similar wave models, as this approach neglects the contribution from the derivative of the wavenumber-dependent weighted current during calculation if the group velocity. BKD17 correct this error and also suggest a strategy for determining the current contribution to group velocity as a function of frequency, employing a Taylor series expansion about the peak frequency, significantly extending the range of accuracy of current information with minimal additional programming or data passage. Here, we extend their results to two horizontal dimensions for use in the SWAN wave model. The expressions for energy density and intrinsic frequency used to construct the wave action density are similarly investigated, using perturbation approximations in the general action balance equation of Voronovich (1976). Approximate wave theory is based on a modification of KC89 assuming strong currents with weak shear. The results suggest that the action density $\mathrm{N}=\mathrm{E}_{0} / \sigma$ may be consistently constructed using the usual expression for energy density, $E_{0}=1 / 2$ $\rho g g a^{2}$, together with a $\sigma=\omega-k \tilde{U}$ based on the KC89 current speed. This result is convenient and adds little complexity to the total amount of information to be passed between NHWAVE and SWAN.

\section{FIELD CASES}

In coastal zones such as river mouths, ambient currents may become strongly sheared due to stratification and tidal effects. For example, the Columbia River ebb-tidal plume has been extensively studied, with effects of turbulence enhancement on frontal development as well as the basic control of wave breaking by blocking on opposing currents drawing recent attention (Thomson et al., 2014; Zippel \& Thomson, 2017) Results from this experiment and others will be used to test the occurrence of blocking effects in the model, examine the evolution of shoaled wave heights, and aid in the parameterization of breaking -induced turbulence levels near the waveaveraged surface layer of the NHWAVE model.

\section{REFERENCES}

Banihashemi, Kirby, Dong (2017). Approximation of the wave action flux velocity in strongly sheared mean flows, Ocean Modeling, 116, 33-47.

Dong (2016): Wave-current interaction in strongly sheared mean flows. University of Delaware PhD thesis. Kirby, Chen (1989): Surface waves on vertically sheared flows: Approximate dispersion relations, Journal of Geophysical Research, 94, 1013-1027.

Ma, Shi, Kirby (2012): Shock-capturing non-hydrostatic model for fully dispersive surface wave processes, Ocean Modelling, 43-44, 22-35.

McWilliams, Restrepo, Lane (2004): An asymptotic theory for the interaction of waves and currents in coastal waters, Journal of Fluid Mechanics, 511, 135-178.

Thomson, Horner-Devine, Zippel, Rusch, Geyer (2014): Wave breaking turbulence at the offshore front of the Columbia River Plume. Geophysical Research Letters. 41, 8987-8993.

Voronovich (1976): Propagation of internal and surface gravity waves in the approximation of geometrical optics. Izv. Atmos. Oceanic. Phys, 12, 850-857.

Zippel, Thomson (2017): Surface wave breaking over sheared currents: Observations from the Mouth of the Columbia River. J. Geophys. Res: Oceans, 122, 33113328. 